\title{
Investigation of Jet Mixing Characteristics Using Slotted Rectangular Tabs
}

\author{
Anusindhiya Krishnaraj' (1D, Vinayagamurthy Ganesan², * (D)
}

1.Vellore Institute of Technology - School of Mechanical Engineering - Chennai/Tamil Nadu - India. 2.Vellore Institute of Technology - Center for Innovation and Product Development - Chennai/Tamil Nadu - India.

*Corresponding author: vinayagamurthy.g@vit.ac.in

\begin{abstract}
This article discusses the jet mixing effectiveness of rectangular tab with slots to distort the jet, thereby creating vortices of differing sizes to enhance the mixing. This article numerically investigates the effectiveness of slanted slotted/perforated rectangular tabs with the slot connecting the adjacent faces of the tab to control Mach 0.4 jet. To intensify the jet mixing process, two similar tabs with equal blockage ratio of $7 \%$ are placed at diametrically opposite locations of the convergent nozzle exit. The jet flow development in the axial and radial directions of the jet are investigated in detail. To measure the effectiveness of the slotted tabs, the results are compared with the free jet and with the jet operated with solid rectangular tab. The percentage reduction in the core length obtained is 84.1 and $74 \%$ for the jet employed with the slotted tab and the solid tab, respectively, when compared with the free jet. The results exhibit that the slotted tab distorts the jet in the near field to a greater extent when compared with the solid rectangular tab.
\end{abstract}

Keywords: Numerical; Core; Distortion; Control; Effectiveness; Vortices.

\section{INTRODUCTION}

With due reference to the basics of the jet theory, it can be contemplated that control of jets and jet mixing becomes necessary for its extensive applications ranging from household appliances to modernistic high technology rockets. Jet mixing plays a vital role in: (i) reducing the jet associated noise produced by the aircraft, (ii) improving the combustor performance, (iii) controlling thrust and (iv) changing the rocket directions (thrust vectoring) etc. Techniques of jet flow control are basically two types - the passive and the active control. Active control of jets involves using external power sources. Passive control of jets includes usage of cross wires, grooved nozzles, mechanical tabs, co-flow nozzles, splitter plates, etc. The usage of cross wires that run diametrically at the exit plain of the nozzle are used in flow control (Lovaraju and Rathakrishnan 2006; Lovaraju et al. 2006). Grooved and chevron nozzles are used as certain techniques in jet flow control (Elangovan and Rathakrishnan 2004; Tide and Srinivasan 2010; Kumar et al. 2011). Nozzles are also modified to issue co-flowing jets to control the flow in the subsonic regime (Lovaraju and Rathakrishnan 2011; Shankar et al. 2016; Shankar et al. 2018; Shankar and Bennett 2019; Thanigaiarasu et al. 2019; Shankar et al. 2020; Shankar and Raja 2020).

The other most effective passive control technique to control the jets is the engagement of mechanical tabs at the exit plane of the nozzle. Passive control of high-speed jets with various tab geometries was studied and documented by researchers.

Received: Jun. 192020 | Accepted: Aug. 182020

Peer Review History: Double Blind Peer Review.

Section Editor: Rho Shin Myong

This is an open access article distributed under the terms of the Creative Commons license. 
With the engagement of tabs that are placed in the direction perpendicular to the mainstream jet flow, counter rotating transverse vortices are generated, which become stream-wise after leaving the tab. It is also a known fact that when the vortices are small, the mixing effectiveness is improved because the small-scale vortices have the capability to travel longer distance along the jet axis and are more stable.

The effects of rectangular tabs in controlling the jet emanating from the convergent nozzle were initially studied by Bradbury and Khadhem (1975). The potential core of the jet can be reduced to a greater extent when the tabs are kept at the exit of the convergent nozzle. The use of mechanical tabs (Ahuja and Brown 1989; Bohl and Foss 1996; Wishart et al. 1993; Zaman et al. 1994) quantifies in controlling the jet and the formation of paired vortices, which are counter rotating in the streamwise direction and their effect on jet mixing and jet spread.

Singh and Rathakrishnan (2002) studied the use of rectangular tabs for sonic jets. The tabs lead to rapid mixing at the centerline and were also found to weaken the shocks by providing a reduction of about $80 \%$. Clement and Rathakrishnan (2006), studied jets from a sonic nozzle operated at nozzle pressure ratio of 2 to 7 . The blockage ratio of the tabs is maintained at $10.18 \%$ and the length-to-width ratio of the tabs is investigated. Weakening of shock waves was observed along with reduction of core of about $75 \%$.

The mechanical tabs of rectangular geometry were then modified by researchers for the enhancement in jet mixing. Then, the use of arc tabs came into being. Two configurations of arc tabs facing in and facing out were used to control the jets. The under expanded sonic jet was analyzed by Thanigaiarasu et al. (2008) and was thus concluded that the arc tab facing in had an $80 \%$ reduction in core length while compared to that of just $40 \%$ in arc tab facing out. Straight perforation on arc tabs greatly influences in jet control by the enhancement of mixing characteristics (Dharmahinder et al. 2011). The implementation of tabs with perforations/slots paved the way for further advancements in the jet flow control, as the slots are known to reduce the thrust losses and effectively reduce blockages. A rectangular tab with slanted perforations, with perforations connecting the opposite edges, are found to be equally competent in promoting the jet mixing process (Ahmed et al. 2013). To further bring in to the advancements, change of profiles of perforations was analyzed by Ahmed et al. (2015), at 30 slant angle and reported that the tab with circular perforation is more efficient in distorting the jet. As the jet flow control differs concerning subsonic and supersonic regimes and flow conditions, Ezhilmaran et al. (2018) attempted to experimentally compare the effect of straight and slanted perforations on the rectangular tab to control the supersonic jet of Mach 1.8. The effectiveness of straight, perforated tab and a slanted perforated tab is studied by Ezhilmaran et al. (2019) by varying the perforation diameter to control Mach 1.5 jet.

Research on mechanical tabs with various other shapes and geometries was done by authors subsequently. One such shape transformation includes the usage of triangular tabs and truncated triangular tabs. These tabs were also given corrugations on the slanting edges of the tabs for reducing thrust losses. Corrugations in the form of semicircle, triangle and square on the sides of the triangular tabs were placed at certain locations and analyzed by Kumar and Rathakrishnan (2013), out of which the tab with semicircular corrugation were found to be better performers for promoting mixing of Mach 2 Jet. A reduction of $90 \%$ in the presence of marginally adverse pressure gradient, in comparison to uncontrolled jet was achieved for the flow corresponding to a nozzle pressure ratio of 7 . The authors then came up with truncated triangular right-angled tabs, which also had corrugations of different shapes and studied the same at Mach 2 (Kumar and Rathakrishnan 2015). Further, the authors concluded that the jet mixing is influenced by the tab geometries, tip effects, corrugation shapes and expansion ratios.

The locations/placements of the corrugations on a triangular tab were also varied and studied numerically and experimentally by researchers in the recent times. Numerical comparison of the semicircular corrugated triangular tab with plain triangular tab to control the subsonic jet is investigated by Kumar and Chidambaram (2018), who found that the tab with corrugations yielded better effectiveness. Investigations by Kumar and Chidambaram (2019) on the corrugation locations by differing the spacing of the locations on the triangular tab to control the supersonic jet yielded more accurate results in this domain. 
From the works of literature and previous researches, it is evident that the perforated tab distorts the jet to increase the jet mixing to a greater extent and it is also clear from the previous researches that perforations or slots were only made in between the opposite faces of the rectangular tabs to control the subsonic and supersonic jet. This study is substantially essential, as it is focused on the analysis of a rectangular tab with the slot connecting the adjacent faces to control the subsonic jet.

For a secondary jet, which intersects the mainstream at a certain inclined angle, the effectiveness improves by controlling the secondary jet from the perforations to impinge into the mainstream at an effective intersection point, which is within the potential core length (Ahmed et al. 2013). Therefore, in this article, the rectangular tab with the slot connecting the adjacent sides of the rectangular profile is investigated to improvise the above explained flow phenomenon. Thereby, the secondary jet from the slot is amended and redirected to hit the mainstream to impinge the primary jet at an inclination. The secondary jet is amended in such a way that it directly hits the core of the jet, by that way it creates high turbulence, hence, higher jet mixing. The vortices formed by the slotted tab are of mixed sizes due to the secondary jet impinging out from the slot provided on the rectangular tabs. The mixing effectiveness of the slotted tab is examined in the presence of zero pressure gradient and it is compared with the plain rectangular tabbed jet and free jet. The blockage ratio of both rectangular slotted tab and solid rectangular tabs are about $7 \%$. To investigate upon the jet flow development and jet spread characteristics, a numerical study is carried out for Mach 0.4 and the results are obtained along the jet centerline and normal to the tab axis at different axial locations of the jet. The experiment is also done for the jet employed with slotted rectangular tab to validate and reinforce the numerical results.

\section{ANALYSIS OF THE SLOTTED TABS}

\section{Design Parameters of Nozzle and Tabs}

A convergent nozzle with $15 \mathrm{~mm}$ exit diameter is used to examine the mixing effectiveness of the rectangular slotted tab. Two slotted rectangular tabs with flow blocking ratio of about 7\% were kept diametrically opposite sides at the exit plane of the convergent nozzle. The blockage ratio can be calculated by using the nozzle and tab dimensions and it is given by Eq. (1).

$$
\mathrm{BR}=\frac{(2 \mathrm{x} \text { tab area })}{\text { nozzle exit area }}
$$

The length, width and height of the slotted rectangular tab are 4mm, 2 and $4 \mathrm{~mm}$ respectively (Fig. 1). The diameter of the slot connecting the adjacent side of the rectangular portion is $1.5 \mathrm{~mm}$. For comparing the effectiveness of the slotted tab to maintain similar flow blockage ratio, solid rectangular tab with dimensions $4 \times 1.5 \times 4 \mathrm{~mm}$ is chosen for this research. The schematic representation of the convergent nozzle exit with tabs placed diametrically opposite side and the slotted tab is shown in Fig 1.

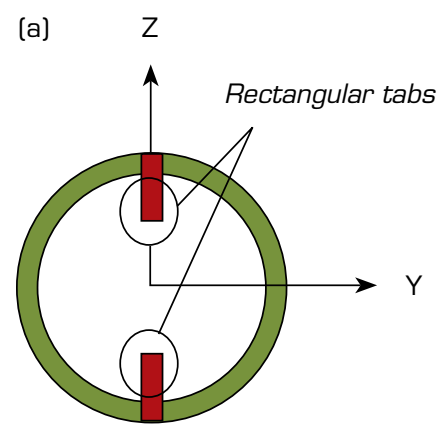

(b)

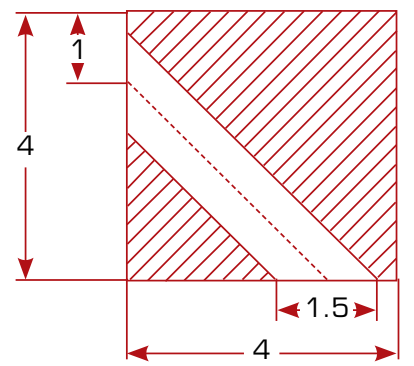

Figure 1. Schematic of (a) Nozzle exit with tabs placed diametrically opposite; (b) Slotted tab used in the present study. 


\section{Details of Experimental Facility Used for Validation}

The experimental setup used to test the tabbed nozzle consists of an open jet test facility. The schematic of the same is shown in Fig. 2. Air pressurized from the compressor is let into the settling chamber and is regulated by a regulating valve. The settling chamber has screen meshes to maintain uniformity and to reduce turbulence at the nozzle inlet. The settling chamber temperature is maintained at that of atmospheric and the flow is made to discharge. The Pitot probe used to measure the pressure at the outlet has an inner diameter of $0.8 \mathrm{~mm}$ and is fixed to a $3 \mathrm{D}$ traverse mechanism. The probe measures pressures along the centerline of the issuing jet. The probe is in turn connected to the data acquisition system. The requisites are then transferred to the computer for further processing.

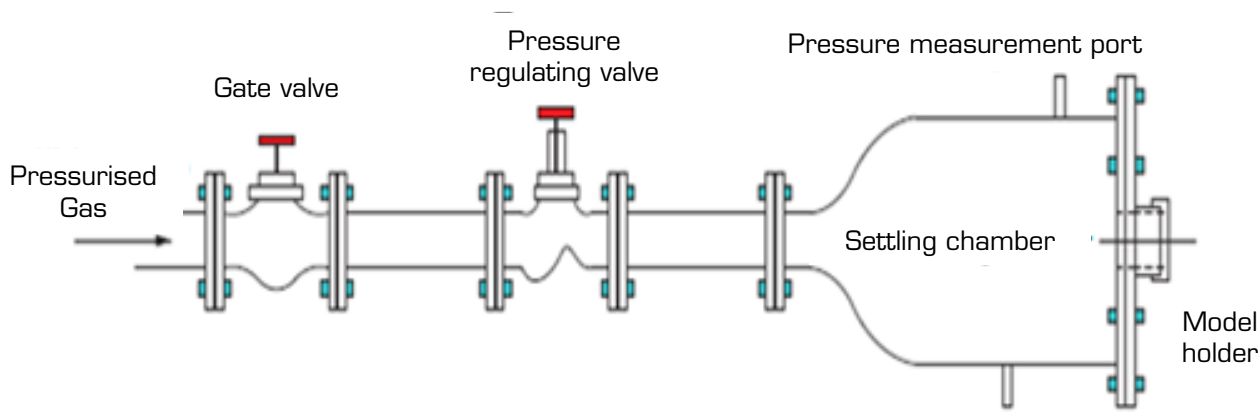

Temperature measurement port

Figure 2. Schematic of the experimental setup used for validation.

The nozzle and the slotted tabs as per the dimensions are fabricated initially using advanced manufacturing technology. The nozzle is fabricated in such a way that it is rigidly fixed with the settling chamber and provisions to fix the slotted tabs to the nozzle is also given by a screwing mechanism. Figure 3 shows the images of the nozzle, along with the tabs used for experiment.
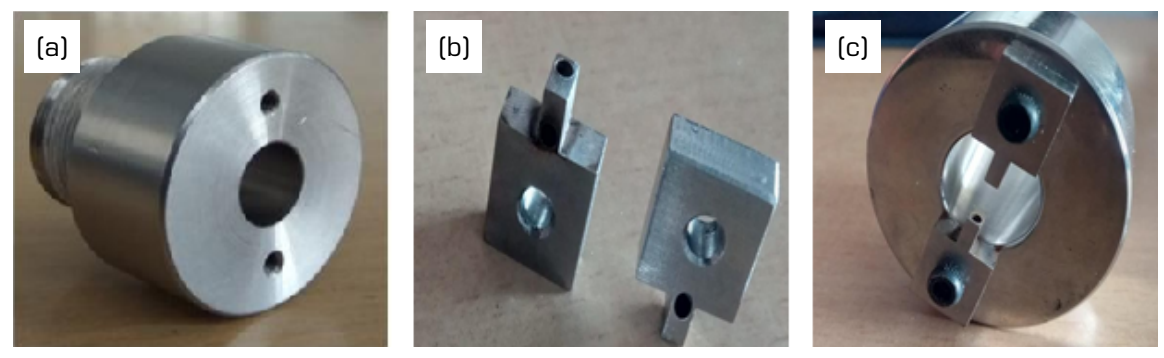

Figure 3. Actual images of (a) Nozzle used for experiment; (b) A pair of slotted tabs; (c) Tabs fitted at the exit of the nozzle.

\section{Computational Domain and Setting Parameters}

To computationally investigate the jet mixing and jet spread characteristics, ICEM CFD and ANSYS CFX softwares are used for generating the grid and for solution solving respectively.

The boundary conditions are initially fixed. The domain of diameter $8 D_{\mathrm{e}}$ (exit diameter) and length $20 D_{\mathrm{e}}$ are used for the study. For inlet, the boundary condition is the pressure inlet of 1.11655 bar corresponding to Mach 0.4 and, for the domain, pressure outlet with the ambient condition is chosen. The temperature is set to $303 \mathrm{~K}$ for the fluid material of air. For the nozzle and tabs wall with the adiabatic, no-slip condition is specified. And the turbulent wall functions for the fluid domain is set to automatic. Figure 4 shows the boundary details of the free jet configuration. The convergence criteria for the variables for solving the solution algorithm is set to $10^{-4}$. 


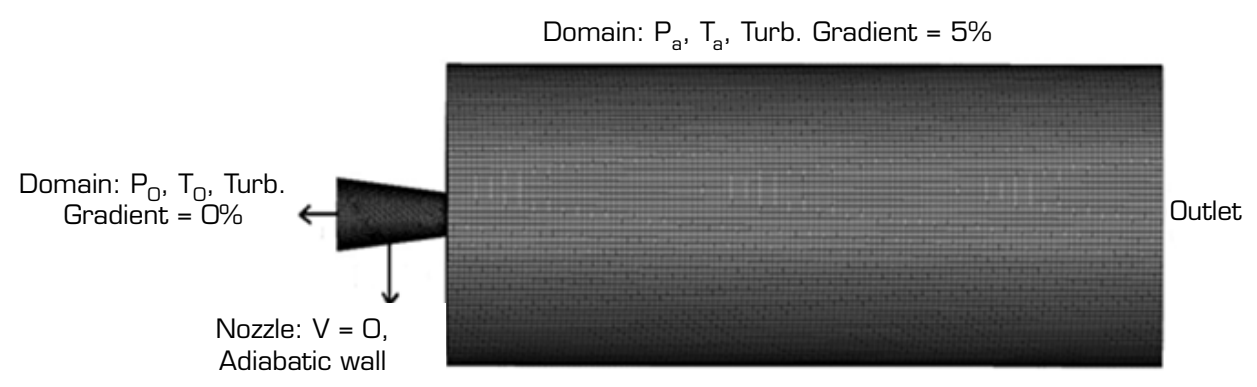

Figure 4. Boundary conditions for the free jet configuration.

\section{Study on Turbulence Models}

Various turbulence models are studied for the investigation of jet with slotted rectangular tab for Mach 0.4. The turbulence models studied were eddy viscosity transport, k-epsilon, BSL Reynolds stress, LRR Reynolds stress, k-omega, QI Reynolds stress, RNG k-epsilon, Shear Stress Transport (SST) and omega Reynolds stress. Figure 5 depicts the results of various turbulence models in comparison with the experiment. From the comparative study using the mentioned turbulence models, SST model is proven to compliment the experimental result.

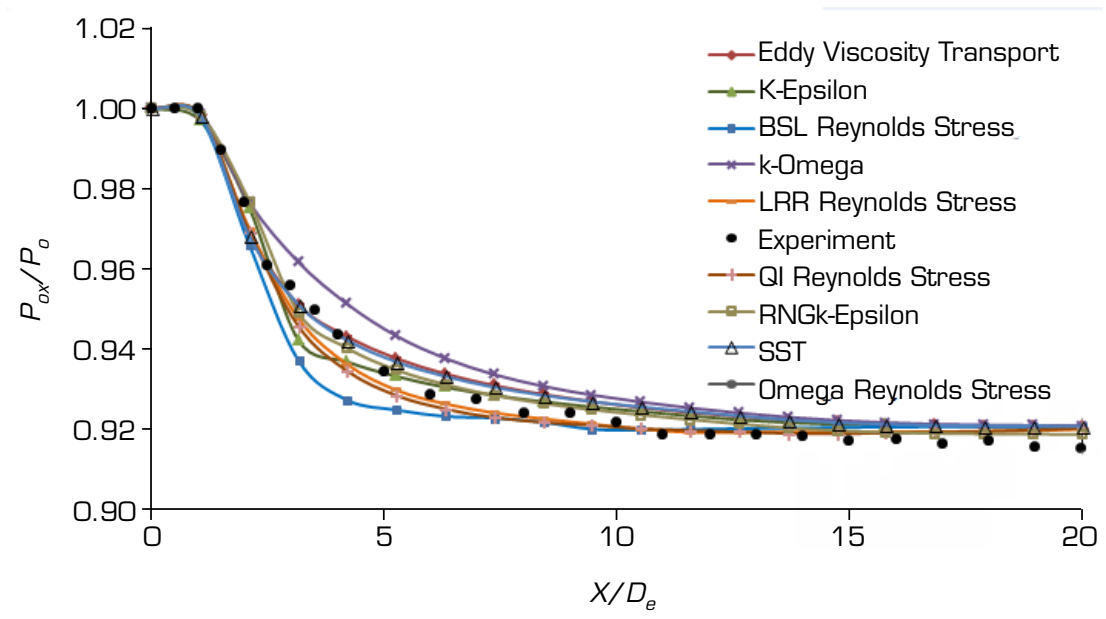

Figure 5. Turbulence model comparison plot for Mach 0.4 jet with slotted rectangular tabs.

The SST turbulence model is one of the effective models used in fluid dynamics for solving a flow. It is a blending function, which blends k-epsilon and k-omega models. The performance of SST is studied and analyzed in many engineering applications. Shear stress transport turbulence model was also used by Kumar and Chidambaram (2018) for the study on triangular tabs with corrugations. The potential core length for the simulation with the SST model and the experiment was as close as 0.87 and $0.88 D_{\mathrm{e}}$, respectively.

\section{Grid Independency Study}

Domain independence and grid independency studies are done to ensure that the results obtained are accurate with no deviations in the obtained results. The domain of diameter 8 and length $20 D_{\mathrm{e}}$ with the tetrahedral mesh of 2.7 million elements were chosen for the study. To capture the jet core accurately and to study near field jet characteristics, the grids are clustered along the jet axis.

The grid independency study carried out for the jet with slotted rectangular tab is shown in Fig 6. Three types of grids namely coarse, medium and fine grids were selected to be used in the study. The results of the numerical analysis are then compared with that of the results of the experiment. Medium grid was chosen for further studies on slotted rectangular tabs. The medium grid was chosen after making alterations in the mesh density, mesh ratio and size of the meshes. A circular region of diameter of about $20 \mathrm{~mm}$ exactly around the centerline of the jet was chosen to have denser meshes, in order to capture the jet behavior in the centerline. And, therefore, the potential core length is determined accurately. 


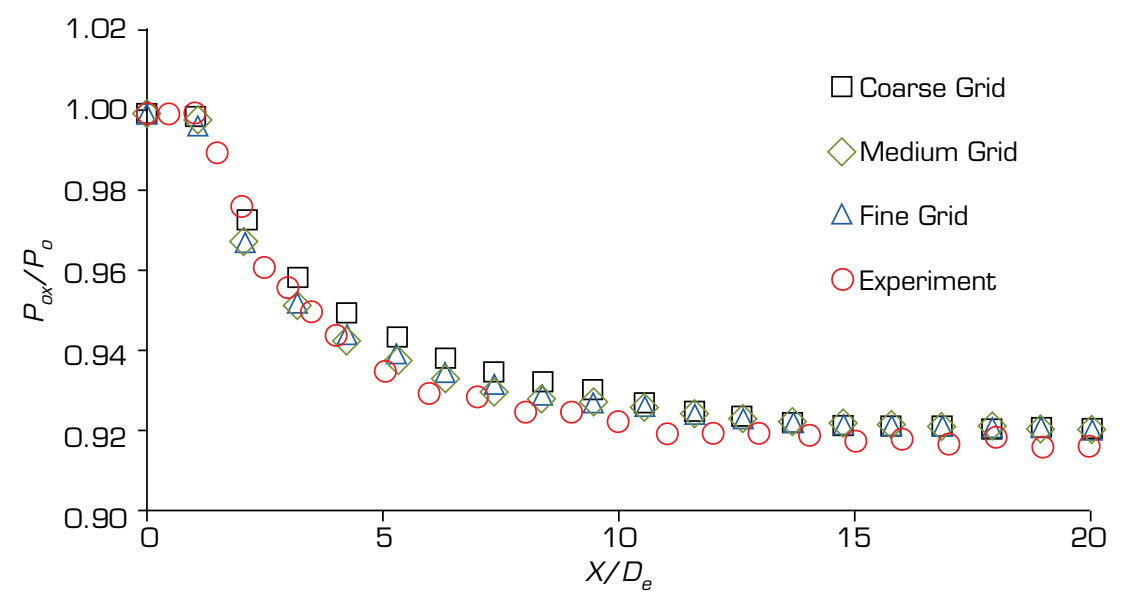

Figure 6. Grid independency study for slotted rectangular tab.

\section{DISCUSSION}

\section{Centerline Decay Profiles (X Direction)}

The jet centerline velocity / Mach number / total pressure decay is a reliable measure of jet mixing (Rathakrishnan 2009), i.e., faster the decay, the faster is the jet mixing with the entrained fluid mass. The centerline velocity decay can clearly state the jet core. The jet core is the distance from the nozzle exit along the centerline, until which the velocity or Mach number or total pressure is unaffected. Figure 7 shows the centerline velocity decay profiles for the free jet, jet employed with solid tab and jet employed with the slotted tab for the jet exit Mach number of 0.4 .

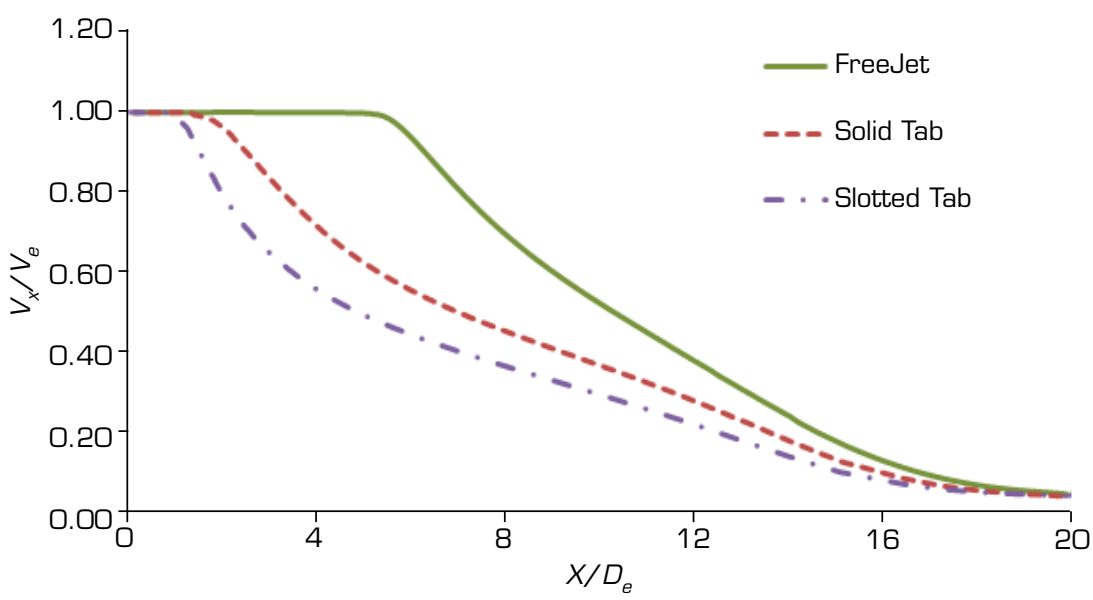

Figure 7. Centerline velocity decay for Mach 0.4 jet.

The total velocity from the exit of the nozzle $\left(V_{\mathrm{x}}\right)$ is measured along the jet centerline for a length of $20 \mathrm{D}_{\mathrm{e}}$. The measured velocity is made dimensionless using the nozzle exit velocity $\left(V_{\mathrm{e}}\right)$ and is plotted as a function of the axial distance $\left(X / D_{\mathrm{e}}\right)$ to investigate the effect of tabs on jet mixing. It is observed that the potential core region lies at 5.16, 1.34 and $0.87 D_{\mathrm{e}}$ for the free jet, solid tab and the slotted tab respectively. Thus, the percentage reduction in the jet core for the solid tab and the slotted tab when compared with the free jet is 74 and $84.1 \%$, respectively. From the profile for the slotted tab, it is observed that the characteristic decay and the self-similar regions were occurring faster than the free jet and jet employed with solid tab. This is due to the combination of counter rotating vortices induced by the tab geometry and the mixed size vortices generated by the jet coming out from the slots of the rectangular tab. The jet coming out from the slots impinges the main flow and distorts the main jet to a considerable amount, leading to the improved mixing. It is evident that the slotted tab changes the jet structure to a more substantial amount than the solid tab to enhance the jet mixing. 


\section{Validation with Experimental Result}

The result of the numerical simulation is validated against the experiment. The validation case study for Mach 0.4 jet enabled with slotted rectangular tab is carried out and the centerline pressure decay plot is extracted. The measured total pressure is nondimensionalized with the jet inlet total pressure and is plotted against the nondimensionalized axial distance $X / D_{\mathrm{e}}$. The results of the numerical simulation and that of the experiment is found to be in good agreement with each other. Figure 8 shows the comparison plot between numerical and experiment.

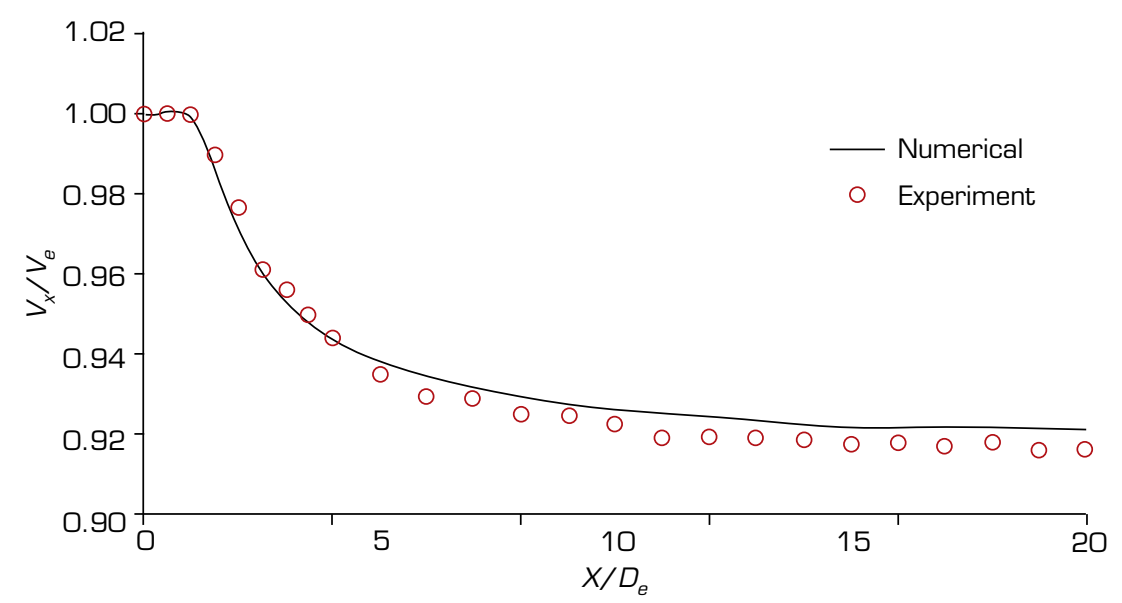

Figure 8. Comparison of the centerline pressure decay plot for Mach 0.4 jet between numerical and experimental results.

\section{Centerline Turbulence Kinetic Energy Profiles}

Turbulence kinetic energy is defined as the mean kinetic energy per unit mass related to the eddies in a turbulent flow. Turbulence kinetic energy has a strong influence on the level of mixing. Figure 9 shows the centerline turbulence kinetic energy profiles of the free jet, solid tab and slotted tab for Mach 0.4 jet. The turbulence kinetic energy from the nozzle exit $\left(k_{\mathrm{x}}\right)$ is measured along the jet centerline and is made dimensionless with the exit turbulence kinetic energy of the jet $\left(k_{\mathrm{e}}\right)$ and is plotted against the axial distance $\left(X / D_{\mathrm{e}}\right)$ to study the jet mixing. The turbulence kinetic energy is almost constant along the core region of the jet (see Fig. 8). And after the core, due to the entrainment of the fluid mass from the ambient (i.e., mixing), the turbulence kinetic energy is increased. The steep rise in the profile indicates that after the rapid mixing process, the turbulence kinetic energy is dropped due to the loss of jet momentum. For the free jet, the ratio $k_{\mathrm{x}} / k_{\mathrm{e}}$ increases steadily after $X / D_{\mathrm{e}}=6$, which means that the turbulence is delayed, hence the mixing capability with the atmosphere is also delayed.

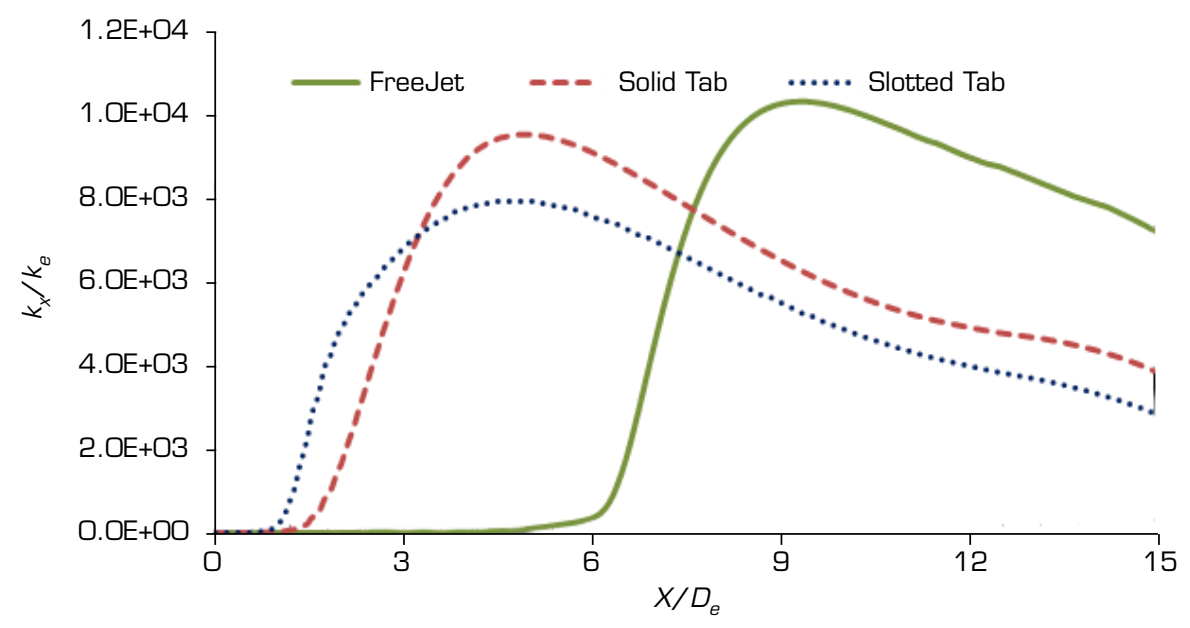

Figure 9. Centerline turbulence kinetic energy profiles for Mach 0.4 jet. 
Whereas for jets enabled with solid tab and slotted tab, it can be interpreted from Fig. 9 that the turbulence intensity increases within the centerline axial distance of $X / D_{\mathrm{e}}=3$. It can be further noted from the figure that, for a jet enabled with slotted tab, the $k_{\mathrm{x}} / k_{\mathrm{e}}$ increases almost at the core and reaches its maximum almost at $X / D_{\mathrm{e}}=4.35$, well before that of the uncontrolled jet and jet with solid tab. Therefore, it can be concluded that there is turbulence in the core region, that is caused by the slotted tab, which leads to faster mixing.

\section{Radial Velocity Profiles Normal to the Tab Axis (Y Direction)}

To get a clear insight into jet spread characteristics, the velocity profiles are studied at locations normal to the tab axis and along the tab axis. The velocity profiles were taken at various axial distances of $0.5,0.8,1,2,4$ and 8 , respectively. The velocity profiles were made dimensionless with reference values of the jet. This following section discusses the radial velocity profiles normal to the tab axis. Figure 10 represents the radial velocity profiles at various $X / D_{\mathrm{e}}$ locations $0.5,0.8,1,2,4$ and 8 for Mach 0.4 jet.

(a)

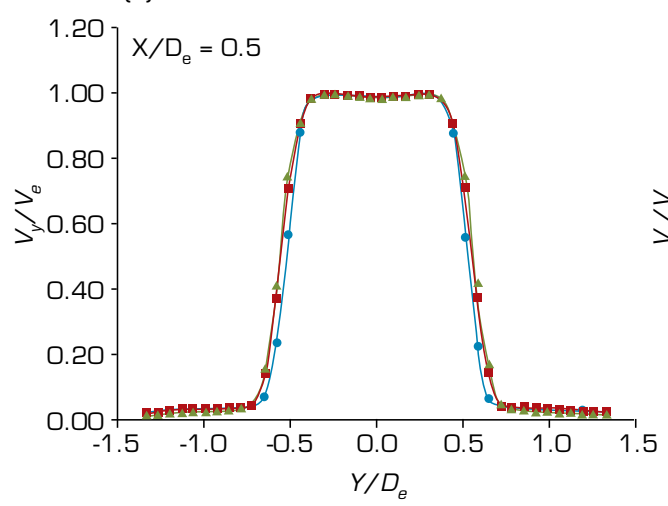

(c)

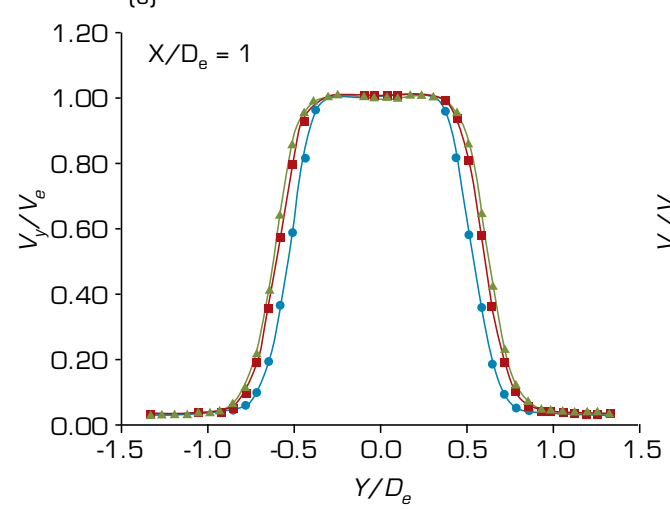

(e)

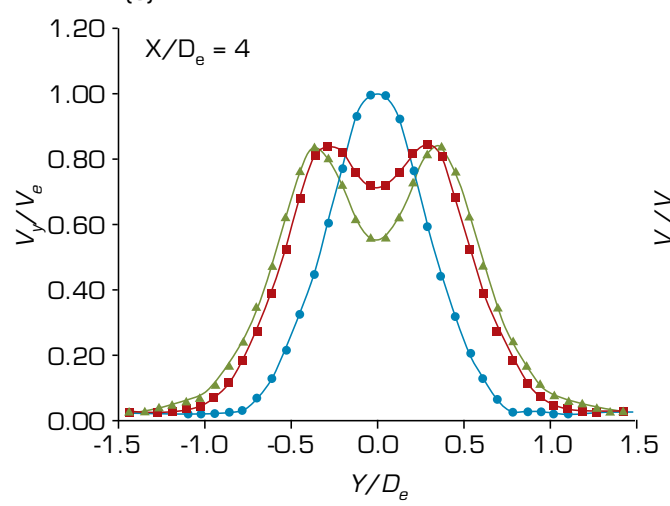

(b)

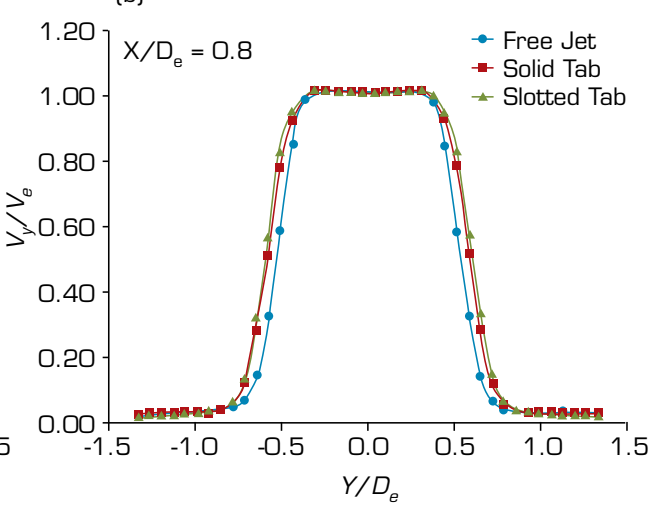

(d)

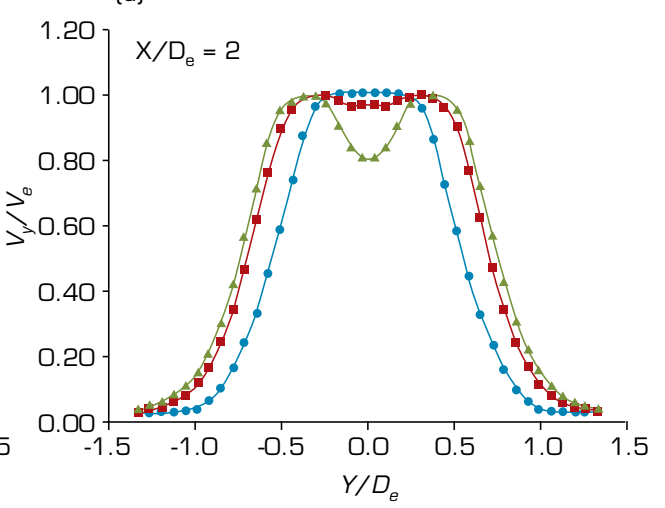

(f)

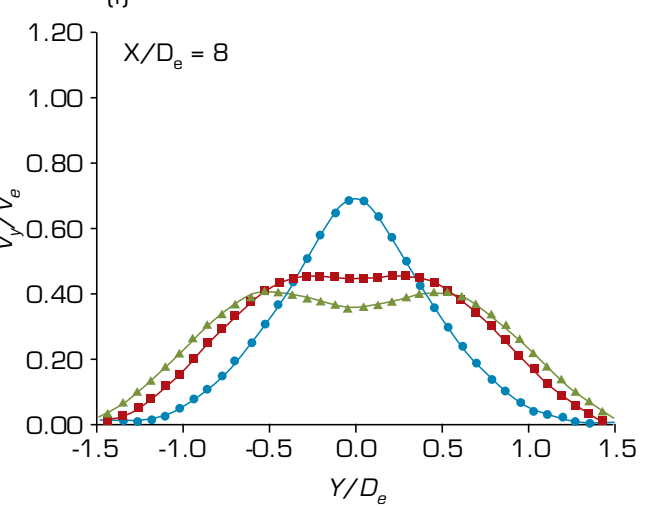

Figure 10. Radial velocity profiles normal to the tab axis at various $X / D_{\mathrm{e}}$ locations. 
The measured radial velocity is made dimensionless with the nozzle exit velocity and is plotted against the nondimensional radial distance $Y / D_{\mathrm{e}}$. At the jet near field locations, i.e., at $X / D_{\mathrm{e}}=0.5,0.8$ and 1 , the velocity profiles form a top hat like structure (Rathakrishnan 2009). As the axial distance is increased, the velocity profiles resemble like a hill with two zones of intense vorticity in the flow on either side of the tab. At $X / D_{\mathrm{e}}=2,4$ and 8 , for the slotted tab employed jet, the minimum velocity ratio exists at $Y / D_{\mathrm{e}}=0$. At $X / D_{\mathrm{e}}=2$, the minimum velocity ratio at $Y / D_{\mathrm{e}}=0$ for the solid tab is 0.96 and for the slotted tab is 0.8 . At $X / D_{\mathrm{e}}=4$, the minimum velocity ratio is 0.72 and 0.56 for the solid and slotted tab employed jet.

Similarly, at $X / D_{\mathrm{e}}=8$, the minimum velocity ratio at $Y / D_{\mathrm{e}}=0$ is observed as 0.45 and 0.36 for the slotted tab and solid tab employed jet. This minimum velocity ratio at $Y / D_{\mathrm{e}}=0$ confirms that the jet decay was occurring faster for the slotted tab employed jet than the solid tab employed jet. It is also observed that the jet spread distance is more for the slotted tab employed jet than free jet and solid tab employed jet. This observation strengthens the results of the jet centerline velocity decay profiles, as well as the experimental total pressure decay profile.

\section{Radial Velocity Profiles Along the Tab Axis (Z -Direction)}

Figure 11 represents the radial velocity profiles along the tab axis at various $X / D_{\mathrm{e}}$ locations $(0.5,0.8,1,2,4$ and 8$)$ for Mach 0.4 jet. The measured radial velocity is made dimensionless with the nozzle exit pressure and is plotted against the nondimensional radial distance $Z / D_{\mathrm{e}}$.

(a)

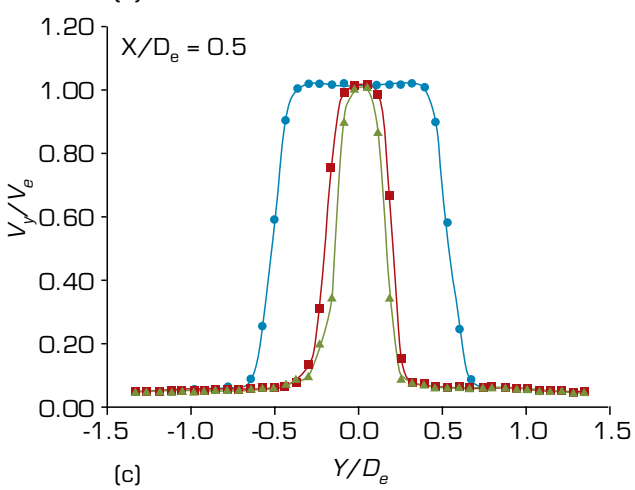

(c)

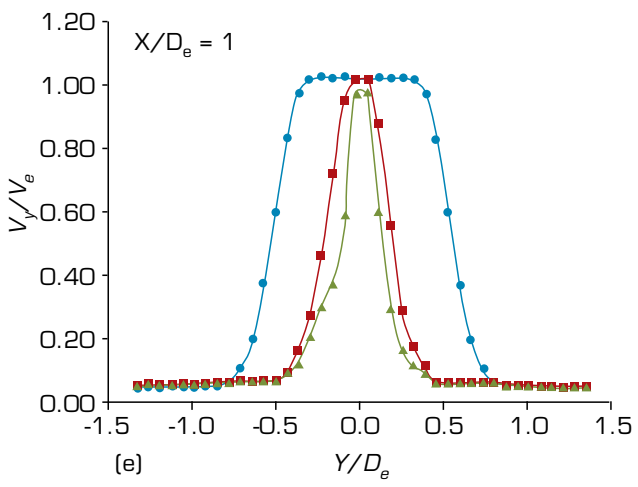

(b)

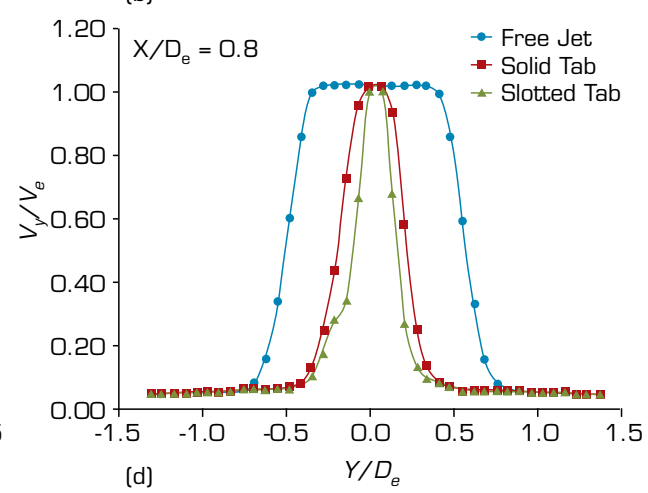

(d)
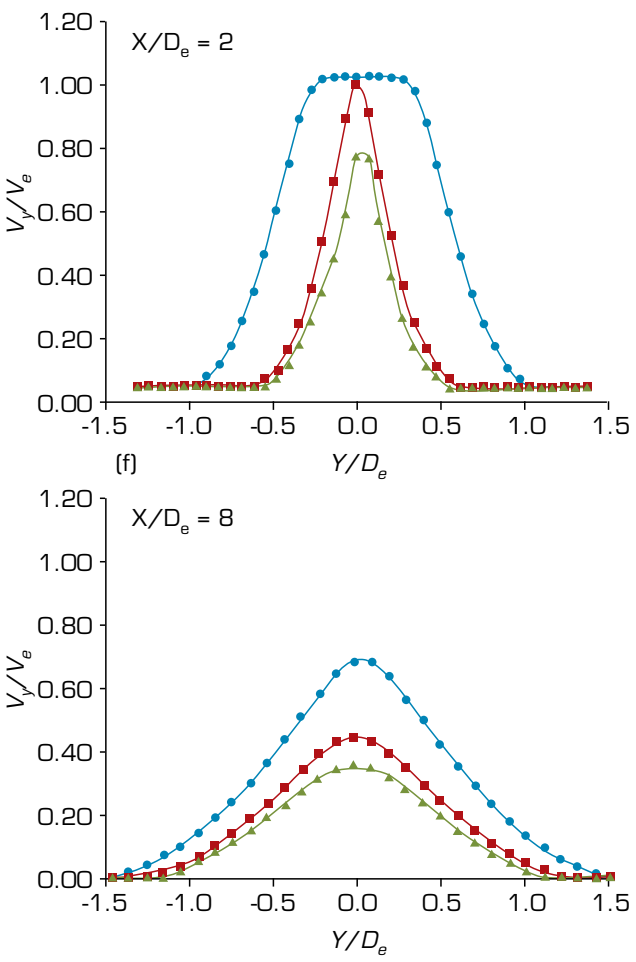

Figure 11. Radial velocity profiles along the tab axis at various $X / D_{\mathrm{e}}$ locations. 
From Fig. 11, it is observed that the jet spread distance for the slotted tab is less when compared to the solid tab employed jet at all axial locations of the jet. This is due to the flow blocking the effect of the tab. At $X / D_{\mathrm{e}}=2,4$ and 8 , the minimum velocity ratio at $Y / D_{\mathrm{e}}=0$ is 0.98 , 0.73 and 0.45 , respectively, for the solid tab employed jet. Whereas for the slotted tab employed jet, the minimum velocity ratio at $Y / D_{\mathrm{e}}$ $=0$ is $0.76,0.54$ and 0.36 at $X / D_{\mathrm{e}}=2,4$ and 8 , respectively. This ensures that the decay is occurring faster for the slotted tab employed jet.

\section{Mach Number Contours in XY Plane}

Figure 12 shows the Mach number contours in the XY plane for all three types of jet. It is seen that the core of the free jet is more when compared with the solid and slotted tab employed jet. When compared to the solid tab, the core of the slotted tab employed jet is less. Along the $y$-direction, the jet spread distance for the slotted tab configuration is more than the solid tab. This ensures that the slotted tab decays faster and enhances the jet mixing process when compared with the free jet and solid rectangular tab employed jet.

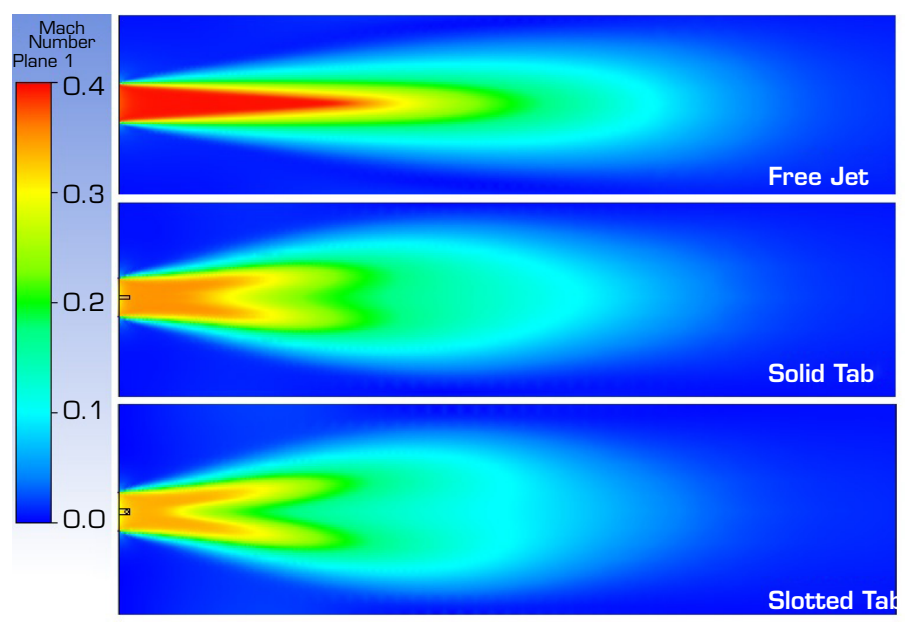

Figure 12. Mach number contour in XY plane.

\section{Mach Number Contours in ZX Plane}

Figure 13 shows the Mach number contours in the ZX plane for the free jet and jet employed with solid and slotted rectangular tab. It is observed that the jet core in this plane is more for the free jet when compared with the solid and slotted tab employed jet. When compared to the solid tab, the jet core of the slotted tab employed jet is less. In this plane, the secondary jet coming out from the slots of the rectangular tab, distorting the main jet is clearly visible, which reduces the core length. Along the $\mathrm{z}$-direction, the jet spread distance for the slotted tab is less when compared with the solid tab, ensuring the faster decay.

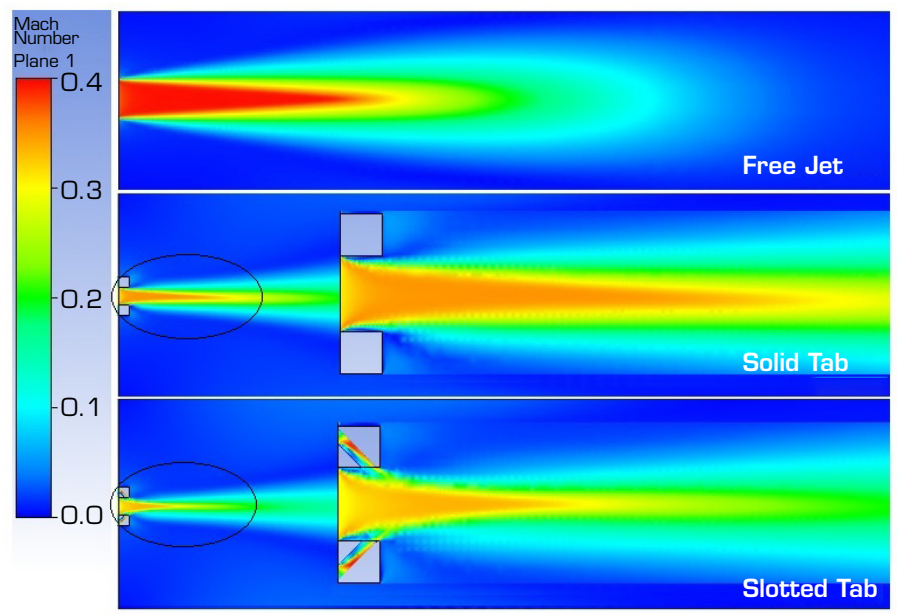

Figure 13. Mach number contours in ZX plane. 


\section{Mach number contours in YZ plane}

Figure 14 shows the Mach number contours at various axial locations $\left(X / D_{\mathrm{e}}=1,2,4\right.$ and 8$)$ of the jet for all the jet types in the YZ plane. At all the axial locations, for the free jet configuration, the contour looks like a concentric cylinder. At $X / D_{\mathrm{e}}=1,2$ and 4 , the contour of the solid and slotted tab resembles like a dumbbell. At $X / D_{\mathrm{e}}=1$ and 2, the slotted tab employed jet has a strong influence in distorting the jet and forms a thin neck at the center when compared with solid tab employed jet.

(a)

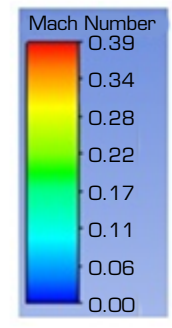

(b)
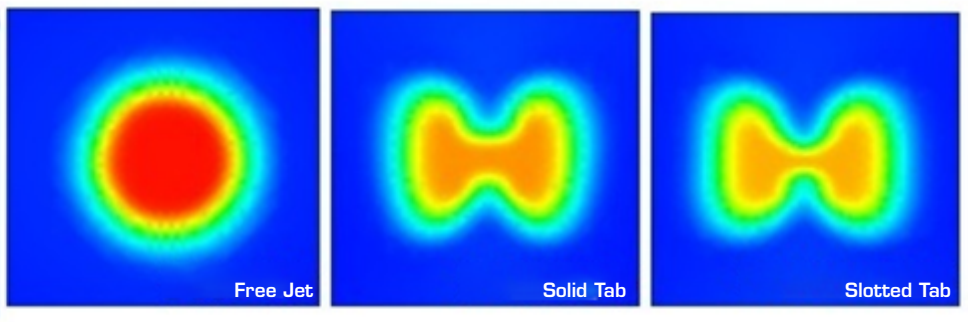

$$
X / D_{e}=1
$$
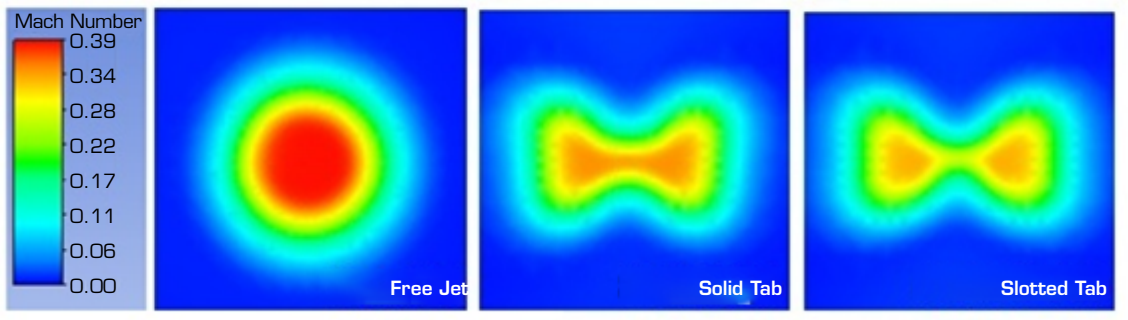

(c)

$$
X / D_{e}=2
$$
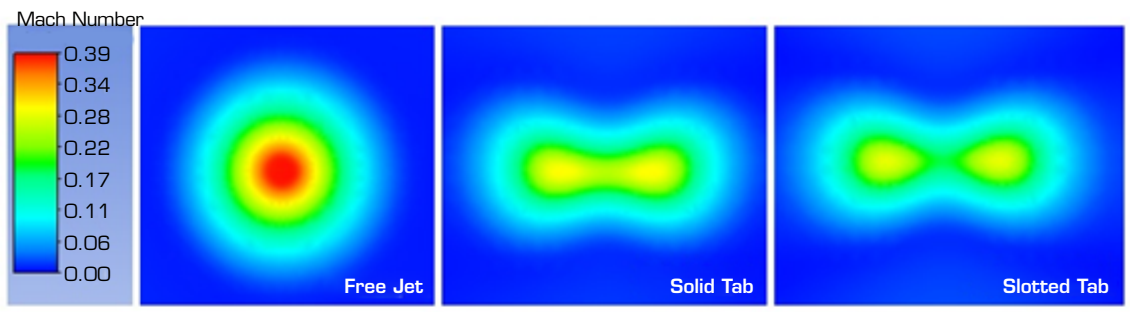

[C]

$$
X / D_{e}=4
$$
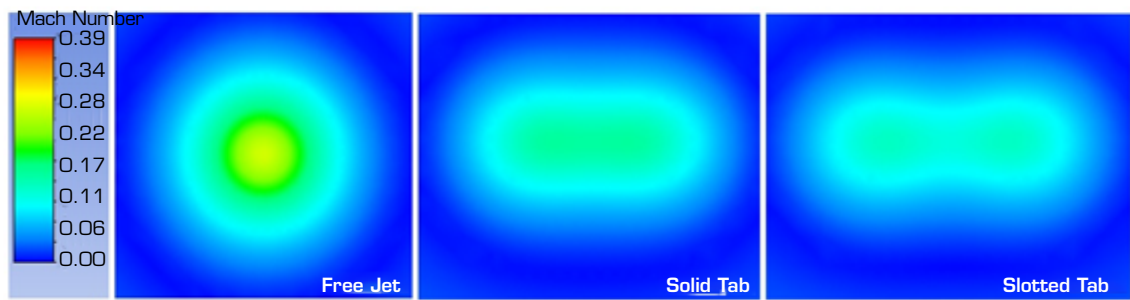

$$
X / D_{e}=8
$$

Figure 14. Mach number contours in YZ plane.

At $X / D_{\mathrm{e}}=2$ and 4, due to intense vortices, the jet bifurcates and forms two velocity zones on either side of the tab, seen in the contour. This reinforces and augments the results of the radial velocity profiles in $y$-direction. At $X / D_{\mathrm{e}}=8$, the contour of the solid tab bears resemblances to an oval, whereas for the jet employed with slotted tab, loses the velocity to a greater extent. 


\section{CONCLUSIONS}

The effectiveness of the rectangular tab with slot connecting the adjacent faces is studied for the jet exit Mach number 0.4 and is compared with the free jet and the jet employed with solid rectangular tab. The centerline decay profile exhibits that the jet with slotted perforated tab enhances the jet mixing process than the solid rectangular tabbed jet. The percentage reduction in core length obtained for the slotted tab configuration is $84.1 \%$, whereas for the solid tab employed jet, the core reduction is $74 \%$ when compared with the free jet. The results of the radial velocity profiles also exhibit that the jet spread characteristics of the slotted tab employed jet is better than the solid tab employed jet. The Mach number contours extracted by cutting out various planes of the jet gives a brief insight about the jet flow development and its structure at various $X / D_{\mathrm{e}}$ locations of the jet. Hence from the results of the centerline and radial velocity profiles, it is strongly concluded that the slotted tab with slot connecting the adjacent faces of the rectangular tab proves to be more effective in distorting the jet at the near field and enhances the jet mixing process.

\section{AUTHORS' CONTRIBUTION}

Conceptualization: Anusindhiya K and Vinayagamurthy G.; Methodology: Anusindhiya K; Investigation: Anusindhiya K and Vinayagamurthy G.; Writing - Original Draft: Anusindhiya K; Writing - Review and Editing: Anusindhiya K and Vinayagamurthy G.; Supervision: Vinayagamurthy G.

\section{DATA AVAILABILITY STATEMENT}

The data sets supporting the conclusion of this article are included in this article.

\section{FUNDING}

Not applicable.

\section{ACKNOWLEDGEMENTS}

We sincerely express our thanks to Aerodynamics laboratory, VIT Chennai for their unconditional support in conducting the experiments and utilizing the computational facility.

\section{REFERENCES}

Ahmed AAR, Thanigaiarasu S, Venkatraman S, Srinivasan E, Rathakrishnan E (2015) Study of slanted perforated shapes in tabs for control of subsonic Jets. AIAA -2423. 33rd AIAA Applied Aerodynamics Conference. Dallas, Texas, United States. https://doi.org/10.2514/6.2015-2423

Ahmed RA, Thanigaiarasu S, Santhosh J, Elangovan S, Rathakrishnan E (2013) Study of Slanted Perforated Jets. Int J Turbo Jet-Engines 30(4):347-357. https://doi.org/10.1515/tjj-2013-0015 
Ahuja K, Brown W (1989) Shear flow control by mechanical tabs. 2nd Shear Flow Conference. AIAA, Tempe, Arizona, United States. https://doi.org/10.2514/6.1989-994

Bohl D, Foss J (1996) Enhancement of passive mixing tabs by the addition of secondary tabs. Paper presented 34th Aerospace Sciences Meeting and Exhibit. AIAA; Reno, Nevada, United States. https://doi.org/10.2514/6.1996-545

Bradbury LJS, Khadem AH (1975) The distortion of a jet by tabs. J Fluid Mech. 70(4):801-813.

Clement S, Rathakrishnan E. (2006) Characteristics of sonic jets with tabs. Shock Waves 15:219-227. https://doi.org/10.1007/ s00193-006-0017-2

Dharmahinder SC, Thanigaiarasu S, Elangovan S, Rathakrishnan E (2011) Perforated Arc-Tabs for Jet Control. Int J Turbo Jet-Engines 28:133-138. https://doi.org/10.1515/tjj.2011.012

Elangovan S, Rathakrishnan E (2004) Studies on high speed jets from nozzles with internal grooves. Aeronaut J 108(1079):4350. https://doi.org/10.1017/S000192400000498X

Ezhilmaran G, Khandai SC, Pavithrabalan S, Udhayakumar K (2018) Supersonic Jet Control by Tabs with Slanted Perforation. Int J Turbo Jet-Engines 1-8. https://doi.org/10.1515/tjj-2018-0034

Ezhilmaran G, Khandai SC, Sinha YK, Thanigaiarasu S (2019) Numerical Simulation of Supersonic Jet Control by Tabs with Slanted Perforation. Int J Turbo Jet-Engines 1-12. https://doi.org/10.1515/tjj-2019-0015

Kumar KS, Chidambaram S (2018) Jet Flow Control Using Semi-Circular Corrugated Tab. Int J Turbo Jet Engines 10:1-13. https://doi.org/10.1515/tjj-2018-0014

Kumar KS, Chidambaram S (2019) Supersonic jet flow control using semi-circular corrugated tabs. Aircr Eng Aerosp Technol 91(10):1340-1352. https://doi.org/10.1108/AEAT-01-2019-0021

Kumar PA, Rathakrishnan E (2013) Corrugated Truncated Triangular Tabs for Supersonic Jet Control. J Fluids Eng 135(9):091104. https://doi.org/10.1115/1.4024204

Kumar PA, Rathakrishnan E (2015) Corrugated right-angled triangular tabs for supersonic jet control. Proc Inst Mech Eng G J Aerosp Eng 229(11):1-9. https://doi.org/10.1115/1.4024204

Kumar PA, Verma SB, Elangovan S (2011) Study of jets from rectangular nozzles with square grooves. Aeronaut J 115(1165):187-196. https://doi.org/10.1017/S0001924000005583

Lovaraju P, Clement S, Rathakrishnan E (2007) Effect of cross-wire and tabs on sonic jet structure. Shock Waves.17:71-83. doi: 10.1007/s00193-007-0092-z.

Lovaraju P, Rathakrishnan E (2006) Subsonic and Transonic Jet Control with Cross-Wire. AIAA J 44(11):2700-2705. https:// doi.org/10.2514/1.17637

Lovaraju P, Rathakrishnan E (2011) Experimental Studies on Co-flowing Subsonic and Sonic Jets. Flow Turbul Combust 87:115-132. https://doi.org/10.1007/s10494-011-9332-5

Rathakrishnan E (2009) Experimental Studies on the Limiting Tab. AIAA J 47(10):2475-2485. https://doi.org/10.2514/1.43790

Shankar RKN, Raja ND (2020) Novel Characteristics of Subsonic Coflowing Jets With Varying Lip Thickness. J Aerosp Technol Manag 12:e2520. https://doi.org/10.5028/jatm.v12.1122

Shankar RN, Bennett KS (2019) Characteristics of a co-flowing jet with varying lip thickness and constant bypass ratio. Aircr Eng Aerosp Technol 91(9):1205-1213. https://doi.org/10.1108/AEAT-01-2019-0007 
Shankar RN, Bennett SK, Rala ND, Kumar KS (2020) Characteristics of a co-flowing jet with varying lip thickness and constant velocity ratio. Aircr Eng Aerosp Technol 92(4):633-644. https://doi.org/10.1108/AEAT-05-2019-0104

Shankar RN, Thanigaiarasu S, Elangovan S, Rathakrishnan E (2018) Co-Flowing Jet Control Using Lip Thickness Variation. Int J Turbo Jet Eng 1-14. https://doi.org/10.1515/tjj-2018-0024

Shankar RN, Thanigaiarasu S, Rathakrishnan E (2016) Numerical Characterization of Lip Thickness on Subsonic and Correctly Expanded Sonic Co-flowing Jets. Trans Jpn Soc Aeronaut Space Sci 59(3):134-141. https://doi.org/10.2322/tjsass.59.134

Singh NK and Rathakrishnan E (2002) Sonic jet control with tabs. International Journal of Turbo and Jet Engines 19(12):107-118. https://doi.org/10.1515/TJJ.2002.19.1-2.107

Thanigaiarasu S, Jayaprakash S, Elangovan S, Rathakrishnan E (2008) Influence of tab geometry and its orientation on underexpanded sonic jets. Proc Inst Mech Eng G J Aerosp Eng 222(3):331-339. https://doi.org/10.1243/09544100JAERO299

Thanigaiarasu S, Shankar RN, Rathakrishnan E (2019) Influence of bypass ratio on subsonic and correctly expanded sonic co-flowing jets with finite lip thickness. Proc Inst Mech Eng G J Aerosp Eng 233(7)2536-2548. https://doi. org/10.1177/0954410018782511

Tide PS, Srinivasan K (2010) Effect of chevron count and penetration on the acoustic characteristics of chevron nozzles. Appl Acoust 71(3):201-220. https://doi.org/10.1016/j.apacoust.2009.08.010

Wishart DP, Krothapalli A, Mungal MG (1993) Supersonic Jet Control Disturbances inside the Nozzle. AIAA Journal. 31(7):1340-1341. https://doi.org/10.2514/3.11773

Zaman, KBMQ, Reeder MF, Samimy M (1994) Control of an axisymmetric jet using vortex generators. Phys Fluids 6(2):778793. https://doi.org/10.1063/1.868316 